\title{
Kean Birch (2018) Neoliberal Bio-Economies? The Co-Construction of Markets and Natures. London: Palgrave MacMillan. 208 pages. ISBN 978-3-319-91424-4
}

\author{
Tomás Carrozza
}

tomascarrozza@gmail.com

We can define this work of Kean Birch in two dimensions. On the one hand, it provides a discussion on one of the emerging issues in the STS field: the nature-culture relationship, and more specifically the nature-market relationship. On the other hand, it represents the synthesis of a large work from the author on the subject.

In a first moment, according to Kean Birch, we need to rethink markets and natures in order to understand the trajectories of public policies on bioeconomy in Canada. Through the co-construction between natures and economies we can understands how each process is related to the other. Thus, the question that runs through the book is: can we truly think of nature separate from the economy?

Through eight chapters, the author seeks to answer this question. However, this inquiry is more than a broad, theoretical concern, aiming at understanding the role of the bioeconomy in mitigation processes against climate change. Canada, with a strong matrix based on natural resources, is currently one of the countries making the greatest efforts to advance a sustainable future.

The author initially outlines and discusses these efforts and the related trajectory in the subject. However, based on the pieces of evidence and the complexity of this topic, he decides to suggest an innovative theoretical approach. Subsequently, the author introduces the reader to a study of political-economic materialities as an approach to socio-technical transitions and environmental economic geography. This approach constitutes, according to the author, the basis for showing how natures and markets are co-constructed.

As a result, the author helps the reader to abandon the dichotomous visions of how the economic system "affects" nature. Neoliberalism, as the current hegemonic economic approach, also needs to be thought in spatial and temporal terms. Truly, to advance in understanding how bioeconomy ideas are placed on the agenda, Birch warns the reader that we have to think about how a "neoliberal" nature exists. Only from this vantage point, we can understand Canadian trajectory on bioeconomy. However, Birch also involves the reader in a specific story. As climate change assumes various material forms, the bioeconomy appears as a narrative, as a way of understanding a sustainable future. The author, then, gently nudges the reader into a convincing reconstruction of how this narrative is materialized. But, mainly how this materialization must be understood from a perspective based on the co-construction between natures and economies.

Birch clarifies that this is a process based on the need to think about a future, which begins from a material base and dialogues with actors and public policies. Subsequently, this process transforms the materiality and derives in a dispute of meanings. Thus, the bioeconomy (in this case in Canada) is a way to respond to these disputes, which inevitably contribute to this co-construction. Different actors put their vision at stake, either as bio-based 
products, in response to substitution for fossil fuels or as a socio-technical transition. The bioeconomy, thus, is foremost a dispute of imaginaries, a search for the construction of meanings and a material basis that may complement them.

However, such a material basis is not a static concept. Different waves of materiality complement, enable or constrain future decisions (Gordillo, 2014). In the Canadian Bioeconomy, the development of R\&D activities, public policies, and technological advances all proceed from the existence of materiality, which in turn legitimizes these speeches.

Previous aspects of materiality, in fact, limit this legitimating process. The consolidation of neoliberalism not only gave rise to previous materialities, such as those associated with fossil fuels. It also gave rise, to a specific extent, to a neoliberal nature. To use Birch's words, "there is no neoliberalism without nature and no nature without neoliberalism" (p.107): when politics expresses wishes, only materiality and nature allow them to take place.

This is how public policy enters the game of co-construction. As with the previous material basis, there also existed legislation that accompanied these processes, which constituted the reality of the current bioeconomy. However, this process ends up advancing in questioning sustainability in the future. When politics does take into account previous experiences, the policies designed end up possibly generating new problems, to the point that they may end up not working at all.

In this journey, which explains very clearly the role of materiality, neoliberal nature and public policy in the co-construction of the bioeconomy, the author raises the unavoidable issue of how to think about a process of transition towards sustainability. Understanding the existence of these previous elements is central since a 'neoliberal nature' enables a transition.

In the process of elaborating transitions, imaginaries come to occupy a central place. The Canadian policy, without defining major national lines, however, relies on specific imaginaries that permeate all levels of government. These imaginaries of sustainability and bioeconomy come from a global narrative but end up materializing at the local level. Therefore, a central task of the actor is to understand these 'politicized' materialities. Understanding them makes it possible to think about the design of (alternative) sustainability policies.

Through this theoretical journey, the reader finally understands the role of advanced biofuels in the bioeconomy approach to transitions. Whilst originally biofuels came to represent the way forward now it becomes necessary to think about the value chains associated with these and see how they open a model of greater sustainability. These new technologies require, Birch warns us, the construction of new natures, neoliberal natures.

Neoliberal natures and the related policy measures that seek a transition to sustainability should be compatible with the financial system. However, Birch states: "The political-economic materialities of advanced biofuels may militate against specific forms of private investment, necessitating more direct involvement of the state in support in their expansion" (p. 183).

By the end of the book, it finally appears clear how this co-construction between markets and nature works. Thanks to the successful functioning, the petroleum-based system continues to be predominant, and also due to a matter of sociotechnical and political configuration Kean Birch considers, though, that it is necessary to work on real alternatives to this production system. Since the natures and markets cannot be conceived independently from each other, any viable alternative needs to reconfigure the market as a materialized process to enable the emergence of real alternatives.

These alternatives, which we could call 'new bioeconomies', have to start from understanding this process of co-construction and move on to subvert the principles that engender current neoliberal natures. Drawing from the cases of organic agriculture and eco-economy, Birch shows how some real alternatives exist and are there to rethink these relationships and enable control of the market. Changing our perspective on what is a market can be central building alternatives.

Finally, this book reminds us of the bioeconomy as a subject within the STS field and about studies on materiality gaining momentum. Kean Birch has done a very remarkable job not only in showing 
a novel and empirically relevant approach but also in presenting how materiality is a gateway to expose STS studies to a dialogic process, which complements and improves the existing debates. Last but not least, this book makes a compel- ling argument to re-think the socio-technical approaches of STS in extended ways, where materiality will be likely to occupy an important part of the future agenda of our field of studies.

\section{References}

Gordillo G R (2014) Rubble: The afterlife of destruction. Durham: Duke university press. 